\title{
Dynamics of Surface Migration in the Weak Corrugation Regime
}

\author{
J. V. Barth,* H. Brune, B. Fischer, J. Weckesser, and K. Kern ${ }^{\dagger}$ \\ Institut de Physique Expérimentale, Ecole Polytechnique Fédérale de Lausanne, CH-1015 Lausanne, Switzerland
} (Received 8 July 1999)

\begin{abstract}
We report a systematic study for metal-on-metal surface migration in the weak corrugation regime, i.e., with migration barriers falling below $\approx 100 \mathrm{meV}$. The migration characteristics are elucidated by variable-temperature scanning tunneling microscopy observations in the 50-200 K temperature range, which are analyzed by means of nucleation theory. The results demonstrate that, upon entering the weak corrugation regime, the dynamics of the systems are characterized by increasingly reduced effective preexponential factors, while Arrhenius behavior prevails.
\end{abstract}

PACS numbers: 68.35.Fx, 61.16.Ch, 68.35.Ja, 82.20.-w

The thermal migration of adsorbed atoms on the periodic lattice of a surface offers an ideal test case to systematically investigate the energetics and dynamics of a rate phenomenon in chemical physics. Surface migration requires the overcoming of an energy barrier $E_{m}$ separating two identical states, a process usually obeying an Arrhenius law [1-3]. In particular, the mobility of atoms on metal surfaces has been extensively studied due to its importance for a variety of processes in science and technology, such as heterogeneous catalysis or epitaxial growth [4-6]. The phenomenology is simple: Atoms or small molecules are preferentially adsorbed at specific highsymmetry sites of the surface periodic atomic lattice, which are separated by energy barriers being generally a mere fraction of the adsorption energy. The lateral mobility of the adspecies is driven by the substrate heat bath providing stochastic excitations whose characteristic energy is given by the Debye temperature $(\sim 25 \mathrm{meV}$ for metals). When the density is low, a two-dimensional random motion of isolated adspecies or tracer diffusion [5] is operational; i.e., adsorbates hop from energy minima to neighboring energy minima. This process can be adequately described in terms of transition state theory (TST) [7], when the thermal energies are much smaller than the migration barrier, i.e., $k_{B} T \ll E_{m}$, and when the coupling of the adspecies to the substrate is sufficiently effective to ensure equilibration between the individual jumps. These conditions normally hold for metallic adsorbates on metal surfaces, and the corresponding tracer diffusion coefficient $D^{*}$ is given by [5]

$$
D^{*}=\frac{1}{4} a^{2} \nu_{0} e^{-\beta E_{m}} \equiv D_{0} e^{-\beta E_{m}},
$$

where $\beta=1 / k_{B} T, a$ is the periodicity of the surface lattice, and $\nu_{0}$ is the attempt frequency, frequently associated with the lateral vibrational mode of the adatom; $D_{0}$ is the preexponential factor.

The situation becomes more intricate when the thermal energy increases such that $k_{B} T \sim E_{m}$ and it is anticipated that TST breaks down, until for $k_{B} T \gg E_{m}$ a free twodimensional Brownian motion of the adatoms is expected, where the diffusivity is directly proportional to the temperature [8]. Another complication may arise when the coupling of the adatom to the substrate is weak or strong. The resulting effect is twofold: On the one hand, a decrease of the attempt frequency is expected, which lowers the mobility [9-11]. On the other hand, for the case of weak coupling, adspecies in the process of migration are deexcited less efficiently and may hop further than just to a neighboring minimum. Such "long jumps" effectively increase the mobility, and the parameter $a^{2}$ in the diffusion Eq. (1) has to be substituted by a mean square jump length $\left\langle l^{2}\right\rangle[12]$.

Very recently, the first experimental observations for the extreme situation of an unrestricted two-dimensional Brownian motion at a surface have been reported: Quasielastic helium atom scattering (QHAS) data indicate that with the $\mathrm{Xe} / \mathrm{Pt}(111)$ system the adatoms at low coverage and $T=105 \mathrm{~K}$ form a fully mobile two-dimensional gas due to an extraordinarily small lateral potential corrugation falling below $10 \mathrm{meV}$ [13]. However, the dynamics of this weak corrugation system could not be elucidated, since the results were obtained at a single temperature with $k_{B} T \approx E_{m}$. In the present Letter, we address this question on the basis of variable temperature scanning tunneling microscopy (STM) observations. As a model case, we discuss the self-diffusion of $\mathrm{Al}$ adatoms on $\mathrm{Al}(111)$, where $E_{m}$ was predicted by ab initio density functional theory calculations to be only $\approx 40 \mathrm{meV}$ [14-16].

The experiments were performed in a standard UHV chamber equipped with a variable temperature STM [17]. The $\mathrm{Al}(111)$ single crystal surface was prepared by cycles of $\mathrm{Ar}^{+}$sputtering $\left(\approx 1 \mu \mathrm{A} / \mathrm{cm}^{2}, 500 \mathrm{eV}, 20 \mathrm{~min}\right)$ at 300 and $800 \mathrm{~K}$ flash annealing followed by slow cooldown. Aluminum was evaporated in situ from a Knudsen cell at background pressures below $5 \times 10^{-10} \mathrm{mbar}$. Auger electron spectroscopy revealed the absence of contaminations for Al films as thick as 10 ML [1 monolayer (ML) corresponds to $1 \mathrm{Al}$ atom per $\mathrm{Al}(111)$ substrate atom]. STM data were acquired in the temperature range $60-180 \mathrm{~K}$ isothermally to or at lower temperatures than deposition. 
The extraordinary small value of the calculated lateral corrugation potential of the $\mathrm{Al} / \mathrm{Al}(111)$ system implies that even at low temperatures the thermal energy is a substantial fraction of the migration barrier $\left(k_{B} T / E_{m} \approx 1 / 5\right.$ at $T=100 \mathrm{~K}$ ). The diffusion characteristics of the $\mathrm{Al}$ adatoms are derived by directly determining the temperature dependence of the nucleation island density $n_{x}$, which is analyzed by nucleation theory. This method has proven to be a powerful means for the elucidation of tracer diffusion at metal surfaces [18-20]. When the temperature is sufficiently low, the only mobile species on the surface is the isolated adatom. Then the dimers are stable and represent immobile nuclei (i.e., the critical cluster size is $i=1$ ). With the present system, the stability of dimers is guaranteed up to elevated temperatures due to the high dimer dissociation energy, determined by ab initio calculations to $0.54 \pm 0.06 \mathrm{eV}$ [21]. Dimer mobility can be similarly excluded for the present temperature range, based on the theoretical value of the dimer migration barrier [21] and on the scaling laws in the presence of cluster diffusion $[22,23]$. The saturation island density is thus solely controlled by the tracer diffusion coefficient and the deposition flux $F$. Then the following scaling law relates $D^{*}$ to $n_{x}$ $[19,24]$ :

$$
n_{x}=0.25\left[\frac{D^{*}}{F}\right]^{-1 / 3}=0.25\left[\frac{F}{D_{0}}\right]^{1 / 3} e^{\beta E_{m} / 3} .
$$

Hence, both migration barrier $E_{m}$ and preexponential factor $D_{0}$ can be obtained by determination of $n_{x}$ at different temperatures. An advantage of this approach is that the density of simultaneously diffusing adatoms on the surface can be kept very small, and thus the tracer diffusion

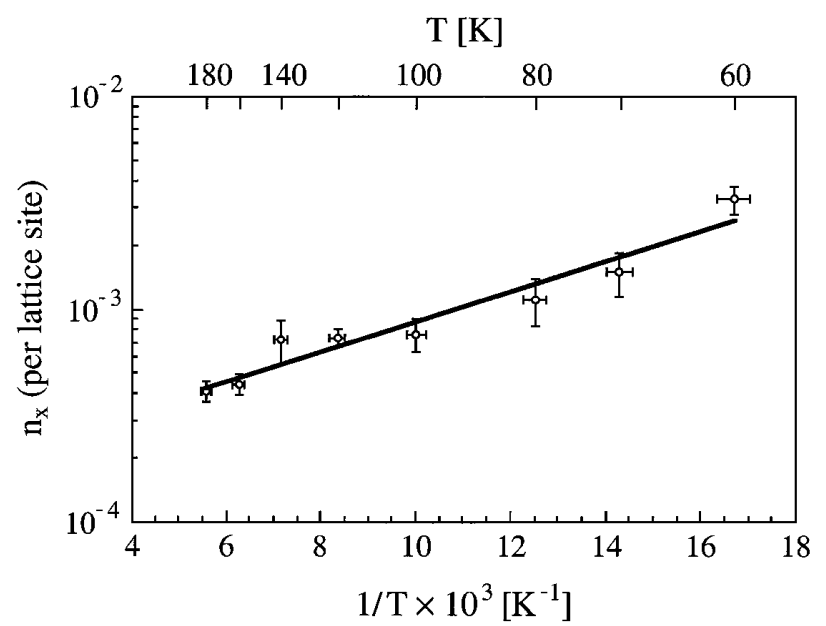

FIG. 1. Arrhenius representation of the saturation island density for Al nucleation on $\mathrm{Al}(111)$ derived from a series of STM images in the temperature range from 60 to $180 \mathrm{~K}(\theta=$ $\left.0.15 \mathrm{ML} ; F=6.2 \times 10^{-4} \mathrm{ML} / \mathrm{s}\right)$. From the slope of the line fit, the migration barrier is determined to be $42 \pm 4 \mathrm{meV}$; the extrapolation for $T \rightarrow \infty$ yields a preexponential factor of $2 \times 10^{-9 \pm 0.25} \mathrm{~cm}^{2} / \mathrm{s}$. of noninteracting adatoms is truly probed. A second advantage is that diffusion and nucleation are not influenced by the presence of the STM tip. Results for the $\mathrm{Al} / \mathrm{Al}(111)$ system are shown by Arrhenius representation for $n_{x}$ reproduced in Fig. 1. The data reveal that $n_{x}$ obeys an Arrhenius law, even if the thermal energies in the individual experiments range from approximately $10 \%$ to $40 \%$ of the expected migration barrier. This clearly indicates that the adatom mobility varies exponentially with the reciprocal temperature in accordance with (1). Thus, (2) can be applied to obtain $E_{m}$, which is determined to $42 \pm 4 \mathrm{meV}$, in very good agreement with the theoretical predictions. By contrast, the corresponding preexponential factor $D_{0}=$ $2 \times 10^{-9 \pm 0.25} \mathrm{~cm}^{-2} / \mathrm{s}$ is exceptionally minute. This value should be compared with the usual magnitude for $D_{0}$ of $\approx 10^{-3} \mathrm{~cm}^{2} / \mathrm{s}$, resulting from the typical dimensions of a surface lattice $(a \approx 3 \AA)$ and the universal TST value of the attempt frequency $\nu_{0} \approx 1 \times 10^{13} \mathrm{~s}^{-1}$ for rate processes at surfaces [25]. Upon assuming that hopping takes place exclusively between neighboring sites, an effective attempt frequency for adsorbed $\mathrm{Al}$ atoms is determined to $\nu_{0}=8 \times 10^{6 \pm 0.25} \mathrm{~s}^{-1}$, i.e., $\nu_{0}$ is 6 orders of magnitude smaller than commonly expected. Note that this calculation of $\nu_{0}$ represents an upper bound for the effective attempt frequency if long jumps were present.

The tremendous decrease of $\nu_{0}$ is associated with the smallness of the migration barrier, which is close to the $\mathrm{Al}$ Debye energy ( $37 \mathrm{meV})$, and the magnitude of the thermal energies involved. It is by no means a singular property for the present system. In a recent investigation of the $\mathrm{Al} / \mathrm{Au}(111)$ system, we found Arrhenius behavior with a minute migration barrier $\left(E_{m}=30 \pm 5 \mathrm{meV}\right)$ along with an even more pronounced reduction of the preexponential factor, which was determined to $D_{0} \approx 5 \times$ $10^{-13} \mathrm{~cm}^{2} / \mathrm{s}$ corresponding to an effective attempt frequency of $\nu_{0}=2.4 \times 10^{3} \mathrm{~s}^{-1}$ [26]. Similar, albeit less drastic effects, exist for the migration of Ag on various fcc surfaces $[18,24,27]$, where, with the migration barrier decreasing from $168 \pm 5$ to $60 \pm 10 \mathrm{meV}$, the corresponding preexponential factor drops from the universal value $D_{0}=1.4 \times 10^{-2 \pm 0.3}\left(\nu_{0}=7 \times 10^{13 \pm 0.3} \mathrm{~s}^{-1}\right)$ to $D_{0}=$ $2 \times 10^{-7 \pm 0.6} \mathrm{~cm}^{2} / \mathrm{s}\left(\nu_{0}=1 \times 10^{9 \pm 0.6} \mathrm{~s}^{-1}\right)$. Note that with all these systems dimer stability $(i=1)$ and the absence of cluster mobility were ensured by investigating the onset of coarsening for dimers [24] or by analyzing the island size distributions [26].

Since all investigations were performed under comparable conditions in the temperature range $50-200 \mathrm{~K}$, the results clearly show a systematic downward shift of the effective attempt frequency with shrinking migration barriers. This trend is visualized by the data compilation in Fig. 2, where we plot $\nu_{0}$ as a function of $E_{m}$. In order to put these results into perspective, the data set is complemented by additional field ion microscopy (FIM) and STM-nucleation theory results for metal on fcc metal surface migration. Note the excellent agreement between the 


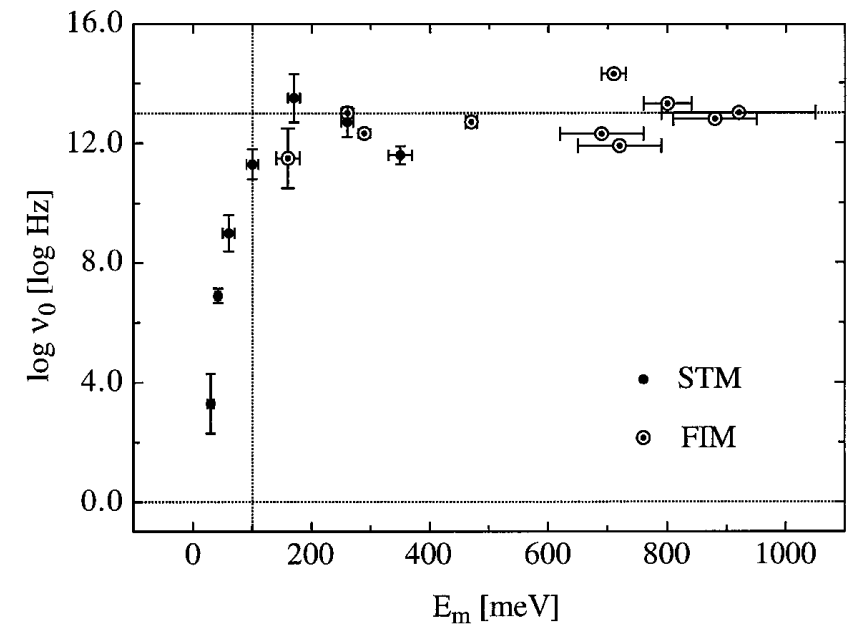

FIG. 2. The effective attempt frequency for intrinsic surface migration of metal atoms on fcc metal surfaces as a function of the migration barrier. In the low-energy regime $\left(E_{m}\right.$ below $\approx 100 \mathrm{meV}$ ) the dynamics of the rate process is characterized by drastically reduced effective attempt frequencies.

STM and FIM derived migration barriers and prefactors with the $\mathrm{Pt} / \mathrm{Pt}(111)$ system $[20,28]$. The corresponding numerical values are comprised in Table I. The compilation reveals that in the weak corrugation limit a novel regime for the dynamics of surface migration is entered, where drastically reduced effective attempt frequencies prevail. This may suggest a breakdown of TST for the present metal-on-metal systems caused by violation of the condition $k_{B} T \ll E_{m}$ with migration barriers falling below a critical value of $\approx 100 \mathrm{meV}$ and simultaneously coming close to the typical excitation energies of the substrate phonon bath. The increasing reduction of $\nu_{0}$ with shrinking migration barriers lends support to this idea.
We note that, as $E_{m}$ decreases, one becomes susceptible also to small lateral interactions between diffusing monomers. Kinetic Monte Carlo simulations show that long-range repulsions ranging over several sites can indeed delay island nucleation which leads to an increase in $n_{x}$ and thus perturbs the scaling law (2) (cf. [35]). However, to explain our observations, these interactions would have to gain importance as $E_{m}$ decreases, whereas the natural expectation would be that also the lateral interactions scale with $E_{m}$. Consequently, the ensemble of our results can hardly be reconciled with failures of density scaling. The data in Fig. 2 can similarly not be described in terms of a compensation effect for phonon-activated Arrhenius processes as suggested in a molecular dynamics study of surface migration on metals [36]. The curved falloff in the approach of the small barriers does not allow for a reasonable fit with the suggested formulas [36], even when exclusively the STM data are considered. This is presumably related to the smallness of the present barriers, coming close to the characteristic excitation energies. Accordingly, the related molecular dynamics simulations reveal an unusual lowering for the effective attempt frequency in this limit [36].

It should be emphasized that our observations are by no means singular, albeit the reported trend could not be extracted thus far due to a lack of systematic observations. On the one hand, the Xe/Pt(111) QHAS observation provides evidence that with the thermal energy approaching $E_{m}$ the nature of the surface migration mechanism undergoes a severe change [13]. On the other hand, related anomalies in the dynamics of surface diffusion with small barriers have been observed earlier by field emission microscopy for adsorbed Xe on W(110) [37,38], direct STM observations for $\mathrm{CO}$ on $\mathrm{Cu}(110)$ [39], and, very recently, also by the integral linear optical diffraction technique for

TABLE I. Characteristics of tracer surface migration for metal atoms on fcc metal surfaces. Effective attempt frequencies were obtained via the relation $D_{0}=(1 / 2 d) a^{2} \nu_{0}$, with $d=1,2$ for one- or two-dimensional diffusion, respectively.

\begin{tabular}{|c|c|c|c|c|c|}
\hline System & $E_{m}[\mathrm{meV}]$ & $D_{0}\left[\mathrm{~cm}^{2} / \mathrm{s}\right]$ & $\nu_{0}\left[\mathrm{~s}^{-1}\right]$ & Method & Ref. \\
\hline $\mathrm{Al} / \mathrm{Au}(111)$ & $30 \pm 5$ & $5 \times 10^{-13}$ & $2 \times 10^{3}$ & STM & [26] \\
\hline $\mathrm{Al} / \mathrm{Al}(111)$ & $42 \pm 4$ & $2 \times 10^{-9 \pm 0.25}$ & $8 \times 10^{6 \pm 0.25}$ & STM & present work \\
\hline $\mathrm{Ag}$ on strained $\operatorname{Ag}(111)$ & $60 \pm 10$ & $2 \times 10^{-7 \pm 0.6}$ & $1 \times 10^{9 \pm 0.6}$ & STM & [27] \\
\hline $\mathrm{Ag} / \operatorname{Ag}(111)$ & $97 \pm 10$ & $4 \times 10^{-5 \pm 0.5}$ & $2 \times 10^{11 \pm 0.5}$ & STM & [27] \\
\hline $\mathrm{Rh} / \mathrm{Rh}(111)$ & $160 \pm 20$ & $5 \times 10^{-5 \pm 1}$ & $3 \times 10^{11 \pm 1}$ & FIM & [29] \\
\hline $\mathrm{Ag} / \mathrm{Pt}(111)$ & $168 \pm 5$ & $1.4 \times 10^{-2 \pm 0.3}$ & $7 \times 10^{13 \pm 0.3}$ & STM & [24] \\
\hline \multirow[t]{2}{*}{$\mathrm{Pt} / \mathrm{Pt}(111)$} & $260 \pm 10$ & $1 \times 10^{-3 \pm 0.5}$ & $5 \times 10^{12 \pm 0.5}$ & STM & {$[20]$} \\
\hline & $260 \pm 3$ & $2.0 \times 10^{-3 \pm 0.15}$ & $1.0 \times 10^{13 \pm 0.15}$ & FIM & [28] \\
\hline $\operatorname{Ir} / \operatorname{Ir}(111)$ & $289 \pm 3$ & $3.5 \times 10^{-4 \pm 0.15}$ & $2.1 \times 10^{12 \pm 0.15}$ & FIM & [28] \\
\hline $\mathrm{Cu} / \mathrm{Ni}(001)$ & $350 \pm 20$ & $6 \times 10^{-5 \pm 0.3}$ & $4 \times 10^{11 \pm 0.3}$ & STM & {$[30]$} \\
\hline $\mathrm{Pt} / \mathrm{Pt}(001)$ & $470 \pm 10$ & $1 \times 10^{-3}$ & $5 \times 10^{12}$ & FIM & [31] \\
\hline $\mathrm{Pt} / \mathrm{Pt}(110)$ in $[001]$ & $690 \pm 70$ & $6 \times 10^{-4}$ & $2 \times 10^{12}$ & FIM & {$[32]$} \\
\hline in [110] & $720 \pm 70$ & $3 \times 10^{-4}$ & $8 \times 10^{11}$ & FIM & [32] \\
\hline $\mathrm{Ir} / \operatorname{Ir}(110)$ in [001] & $710 \pm 20$ & $6 \times 10^{-2}$ & $2 \times 10^{14}$ & FIM & [33] \\
\hline in [110] & $800 \pm 40$ & $4 \times 10^{-3}$ & $2 \times 10^{13}$ & FIM & [33] \\
\hline $\mathrm{Rh} / \mathrm{Rh}(001)$ & $880 \pm 70$ & $1 \times 10^{-3}$ & $6 \times 10^{12}$ & FIM & [29] \\
\hline $\mathrm{Pt} / \mathrm{Rh}(001)$ & $920 \pm 130$ & $2 \times 10^{-3}$ & $1 \times 10^{13}$ & FIM & [34] \\
\hline
\end{tabular}


the system $\mathrm{Xe} / \mathrm{Ni}(111)$ [40]. In all cases, extraordinarily small preexponential factors were encountered.

In conclusion, we investigated surface migration in the weak corrugation regime, where activation barriers approach the thermal and typical phonon excitation energies. It was found that the dynamics of such systems are characterized by increasingly reduced effective preexponential factors the smaller the migration energy barriers encountered. Further theoretical investigations are strongly suggested to clarify the origins of this anomalous diffusion behavior. The universal nature of the underlying physics suggests that such effects may be of general importance for low barrier rate processes driven by thermal activation.

Stimulating discussions with A. Bogicevic, R. Ferrando, K. Fichthorn, and M. Scheffler are gratefully acknowledged.

*Email address: johannes.barth@epfl.ch

${ }^{\dagger}$ Also at Max-Planck-Institut für Festkörperforschung, Heisenbergstraße 1, D-70569 Stuttgart, Germany. Email address: kern@kern.mpi-Stuttgart.mpg.de

[1] I. Langmuir and J. B. Taylor, Phys. Rev. 40, 463 (1932).

[2] E. K. Rideal, Trans. Faraday Soc. 28, 139 (1932).

[3] J. B. Taylor and I. Langmuir, Phys. Rev. 44, 423 (1933).

[4] Surface Mobilities on Solid Materials, edited by V. T. Binh (Plenum, New York, 1983).

[5] R. Gomer, Rep. Prog. Phys. 53, 917 (1990).

[6] Surface Diffusion: Atomistic and Collective Processes, edited by M. C. Tringides (Plenum, New York, 1996).

[7] S. Glasstone, K. J. Laidler, and H. Eyring, The Theory of Rate Processes (McGraw-Hill, New York, 1941).

[8] T. Ala-Nissila and S.C. Ying, Prog. Surf. Sci. 39, 227 (1992).

[9] H. A. Kramers, Physica (Utrecht) 7, 284 (1940).

[10] V. P. Zhdanov, Surf. Sci. 214, 289 (1989).

[11] P. Hänggi, P. Talkner, and M. Borkovec, Rev. Mod. Phys. 62, 251 (1990).

[12] R. Ferrando, R. Spadacini, and G. E. Tommei, Phys. Rev. E 48, 2437 (1993).

[13] J. Ellis, A. P. Graham, and J. P. Toennies, Phys. Rev. Lett. 82, 5072 (1999).
[14] R. Stumpf and M. Scheffler, Phys. Rev. Lett. 72, 254 (1994).

[15] R. Stumpf and M. Scheffler, Phys. Rev. B 53, 4958 (1996).

[16] A. Bogicevic, J. Strömquist, and B. I. Lundqvist, Phys. Rev. Lett. 81, 637 (1998).

[17] H. Brune, H. Röder, K. Bromann, and K. Kern, Thin Solid Films 264, 230 (1995).

[18] H. Brune, H. Röder, C. Boragno, and K. Kern, Phys. Rev. Lett. 73, 1955 (1994).

[19] H. Brune, Surf. Sci. Rep. 31, 121 (1998).

[20] M. Bott, M. Hohage, M. Morgenstern, T. Michely, and G. Comsa, Phys. Rev. Lett. 76, 1304 (1996).

[21] A. Bogicevic, P. Hyldgaard, G. Wahnström, and B.I. Lundqvist, Phys. Rev. Lett. 81, 172 (1998).

[22] S. Liu, L. Bönig, and H. Metiu, Phys. Rev. B 52, 2907 (1995).

[23] M.C. Bartelt, S. Günther, E. Kopatzki, R. J. Behm, and J. W. Evans, Phys. Rev. B 53, 4099 (1996).

[24] H. Brune, G. S. Bales, J. Jacobsen, C. Boragno, and K. Kern, Phys. Rev. B 60, 5991 (1999).

[25] V. P. Zhdanov, Surf. Sci. Rep. 12, 183 (1991).

[26] B. Fischer, H. Brune, J. V. Barth, A. Fricke, and K. Kern, Phys. Rev. Lett. 82, 1732 (1999).

[27] H. Brune, K. Bromann, H. Röder, K. Kern, J. Jacobsen, P. Stoltze, K. Jacobsen, and J. Nørskov, Phys. Rev. B 52, 14380 (1995).

[28] K. Kyuno, A. Gölzhäuser, and G. Ehrlich, Surf. Sci. 397, 191 (1998).

[29] G. Ayrault and G. Ehrlich, J. Chem. Phys. 60, 281 (1974).

[30] B. Müller, B. Fischer, L. Nedelmann, H. Brune, and K. Kern, Phys. Rev. B 54, 17858 (1996).

[31] G. L. Kellogg, Surf. Sci. 246, 31 (1991).

[32] G. L. Kellogg, Surf. Sci. Rep. 21, 1 (1994).

[33] C. L. Chen and T.T. Tsong, Phys. Rev. Lett. 66, 1610 (1991).

[34] G. L. Kellogg, Appl. Surf. Sci. 67, 134 (1993).

[35] K. Fichthorn and M. Scheffler (unpublished).

[36] G. Boisvert, L. J. Lewis, and A. Yelon, Phys. Rev. Lett. 75, 469 (1995).

[37] J.-R. Chen and R. Gomer, Surf. Sci. 94, 456 (1980).

[38] J. R. Banavar, M. H. Cohen, and R. Gomer, Surf. Sci. 107, 113 (1981).

[39] B. G. Briner, M. Doering, K. Rust, and A. M. Bradshaw, Science 278, 257 (1997).

[40] X.-D. Zhu (private communication). 